\title{
Prevalencia de la Giardia duodenalis en el centro de bienestar animal "CEIBA", Rionegro, Colombia (2017)
}

\author{
Prevalence of Giardia duodenalis in the "CEIBA" animal welfare \\ center, Rionegro, Colombia (2017)
}

\section{Prevalência de Giardia duodenalis no centro de apicultura animal de "CEIBA", Rionegro, Colômbia (2017)}

\author{
Vanesa M. Arenas-Angulo, MVZ, MSc ${ }^{1}$; Víctor M. Molina-Díaz, MVZ, MSc ${ }^{1}$
}

1 Programa de Medicina Veterinaria; Facultad de Ciencias Administrativas y Agropecuarias; Corporación Universitaria Lasallista, Caldas, Antioquia, Colombia.

Email: vimolina@lasallistadocentes.edu.co

\section{Resumen}

Determinar la prevalencia de parasitismo de Giardia duodenalis, en el centro de bienestar "CEIBA", del municipio de Rionegro, Colombia durante junio 2017. Se realizó un estudio descriptivo transversal, no experimental, aleatorio, fueron evaluados 112 coprológicos de caninos del centro de bienestar "CEIBA", de ambos sexos, todas las edades y alimentación comercial. Fueron sometidos a evaluación coprológica, por extracción directa de ampolla rectal y evaluada en el Laboratorio Clínico de la Corporación Universitaria Lasallista "Hermano Marco Antonio Serna f.s.c", fueron realizados dos métodos diagnósticos, flotación con solución salina saturada (Willys Molloy) y frotis directo. La presencia de Giardia duodenalis fue categorizado en escala de cruces de 0-3. Se encontraron 60 (55\%) muestras con presencia de parásitos, de los cuales 10 (8,92\%) estaban infestados por Giardia duodenalis, representando 16,66\% de la parasitosis total, para una cruz 2 (20\%), con dos cruces $5(50 \%)$ y con tres $3(30 \%)$. La prevalencia de Giardia duodenalis en el centro de bienestar CEIBA, es baja con respecto a datos de prevalencia en otros albergues o centros caninos, lo cual puede estar influenciado por las medidas de prevención y control de la enfermedad.

Palabras clave: canino, giardiasis, parasitismo, protozoario intestinal.

\begin{abstract}
To determine the prevalence of Giardia duodenalis parasitism at the CEIBA wellness center in the municipality of Rionegro, Colombia, during June 2017. A descriptive cross-sectional, non-experimental, randomized study was carried out. 112 coprological evaluations of canines of the "CEIBA" wellness center of both sexes, all ages and commercial feeding were evaluated. They were submitted to a cochrological evaluation, by direct extraction of rectal ampulla and evaluated in the Clinical Laboratory of the University of Lasallian Hospital "Hno Marco Antonio Serna, fsc", two diagnostic methods were carried out, flotation with saturated saline solution (Willys Molloy) and direct smears. The presence of Giardia duodenalis was categorized as 0-3 crosses. A total of 60 (55\%) samples were present, with $10(8.92 \%)$ infested by Giardia duodenalis, accounting for $16.66 \%$ of the total parasite, for a cross $2(20 \%)$, with two crosses $5(50 \%)$ and three $3(30 \%)$. The prevalen-
\end{abstract}


ce of Giardia duodenalis in the CEIBA well-being center is low with respect to prevalence data in other canines or hostels, which may be influenced by disease prevention and control measures.

Key words: canine, giardiasis, parasitism, intestinal protozoan.

\begin{abstract}
Resumo
Determinar a prevalência do parasitismo de Giardia duodenalis no centro de bem-estar CEIBA no município de Rionegro, Colômbia, em junho de 2017. Foi realizado um estudo descritivo transversal, não experimental, randomizado. Foram avaliadas as avaliações coprológicas dos caninos do centro de bem estar "CEIBA" de ambos os sexos, todasas idades e alimentação comercial. Eles foram submetidos a avaliação cochrológica, por extração direta de ampola retal e avaliada no Laboratório Clínico do Hospital Universitário das Ilhas "Hno Marco Antonio Serna, fsc", foram realizados dois métodos de diagnóstico, flotação com solução salina saturada (Willys Molloy) e esfregaços diretos. A presença de Giardia duodenalis foi categorizada como 0-3 cruzamentos. Um total de 60 (55\%) amostras estavam presentes, com 10 (8,92\%) infestadas por Giardia duodenalis, representando 16,66\% do parasita total, para uma cruz $2(20 \%)$, com duas cruza 5 (50\%) e três 3 (30\%). A prevalência de Giardia duodenalis no centro de bem-estar da CEIBA é baixa em relação aos dados de prevalência em outros caninos ou albergues, o que pode ser influenciado por medidas de prevenção e controle de doenças.
\end{abstract}

Palavras-chave: canino, giardíase, parasitismo, protozoários intestinais.

\section{Introducción}

Los parásitos gastrointestinales son considerados uno de los principales agentes causantes de problemas sanitarios en los albergues caninos (Cabrera y Molina, 2016), las prevalencias descritas son muy variadas y depende de las condiciones de manejo y control, siendo el parasitismo por Giardia duodenalis, uno de los agentes causante de enteritis en caninos con potencial zoonótico (Cabrera y Molina, 2016; Payne y Artzer, 2009; Rodríguez et al., 2014). Cabe anotar que este parásito no solo afecta a los humanos y caninos (Rodríguez et al., 2014, Alarcón, Juyo, Larrotta, 2015), sino también muchas especies como felinos, primates, suinos entre otros (Bouzid et al., 2015; Ivanov, 2010), encontrándose con mayor frecuencia en las grandes ciudades, por su forma de transmisión (Payne y Artzer, 2009). Se han descrito varios genotipos, lo que ha creado confusión sobre las especies, tratándose del mismo agente (Ivanov, 2010).

Este agente protozoario de tipo flagelado, presenta afinidad por la mucosa intestinal, en especial del intestino delgado, en el cual causa lesiones inflamatorias caracterizada por ulceraciones (Bouzid et al., 2015; Caccio et al., 2005; Payne y Artzer, 2009), y cuadros de gastroenteritis (Payne y Artzer, 2009), la presencia de giardia, no implica siempre que los caninos tengan enteritis (Cabrera y Molina, 2016) pero si se comportan como un potencial zoonótico a tener en cuenta desde la salud pública (Kotloff et al., 2013; Rodríguez et al., 2014).

Es bien documentado, el riesgo sanitario de la presencia de giardiasis en las especies domésticas y en humanos (Rodríguez et al., 2014; Meireles et al., 2008;
Oliveira, Amarante, Ferrari, Nunes, 2002), siendo el manejo de aguas residuales un verdadero desafío en el control, en especial en centros de bienestar animal (Caccio et al., 2005; Ferraz et al., 2017; Thompson, 2004). Debido a que las condiciones de hacinamiento y socio sanitarias influyen marcadamente en la presencia del agente como los describe Ferraz et al., (2017).

El diagnóstico como prueba estándar es la determinación de la G. duodenalis en heces (Caraballo et al., 2007; Fernandes de Mendoça et al., 2017), el método descrito para su identificación es el frotis directo, en el cual se determina la presencia de la forma quística o de la presencia de trofozoítos (Cardona et al., 2014; Fernandes de Mendoça et al., 2017; Payne y Artzer, 2009), aunque existen pruebas de inmunofluorescencia, como las descritas por O'Handley et al., (2001), comercialmente y Merifluor ${ }^{\circledR}$ (Cryptosporidium/Giardia kit, Meridian Diagnostics Inc., Cincinnati, $\mathrm{OH}$ ), en la actualidad no se disponen en Colombia (Geurden et al., 2011; O’ Handley et al., 2000) y la técnica de frotis directo es aceptada para el diagnóstico de G. duodenalis en Colombia (Rodríguez et al., 2014; Jiménez et al., 1994).

No existen estudios de prevalencia de G. duodenalis en caninos en Colombia, pues los datos existentes son de pequeños estudios descriptivos como los hechos por Cabrera y Molina (2016), Alarcón et al., (2015), Caraballo et al., (2007) y Sierra-Cifuentes et al., (2015), donde describen frecuencias desde 0.80-54.50\% respectivamente. A nivel mundial se estima una prevalencia del $7 \%$ y en Suramérica se ha descrito una prevalencia del 27.6\% (Bouzid et al., 2015). El objetivo de este estudio fue determinar la prevalencia de G. duodenalis en el centro de bienestar animal "CEIBA", 
de la alcaldía de Rionegro, Antioquia, Colombia, como parte del programa de salud pública del programa de Medicina Veterinaria de la Corporación Universitaria Lasallista.

\section{Materiales y métodos}

\section{Tipo de estudio}

Se realizó un estudio descriptivo transversal, no experimental, realizado en junio 2017.

\section{Localización}

El estudio fue realizado en el municipio de Rionegro, Antioquia, Colombia, en el Centro de Bienestar Animal "CEIBA", ubicado a $6^{\circ} 09^{\prime} 12^{\prime \prime} \mathrm{N} 75^{\circ} 22^{\prime} 27^{\prime \prime} \mathrm{O}$, en los $2080 \mathrm{msnm}$ y con temperatura promedio $18.5^{\circ} \mathrm{C}$.

\section{Población}

Fueron realizados muestreo de materia fecal de 122 caninos, incluyendo ambos sexos y cualquier edad, todos los animales se encuentran en las mismas condiciones climáticas, de alojamiento y alimentación, los criterios de inclusión fueron el $100 \%$ de la población y el criterio de exclusión fue la presencia de una enfermedad concomitante, lo cual para el presente estudio fue del $0 \%$.

\section{Aspectos éticos}

Las actividades realizadas fueron siguiendo los protocolos sanitarios de control de parasitismo interno, como parte del compromiso de evaluación sanitaria, de los caninos albergados en el centro y como componente de la vigilancia sanitaria de los mismos, ningún animal fue sometido actos por fuera de la Ley 576 del 2000, capítulo 3 y 6, Ley 84 de 1989 y Ley 1774 de 2016 del congreso Nacional de Colombia. La toma de muestras en pacientes hace parte de las funciones del convenio interinstitucional suscrito entre la alcaldía de Rionegro y Corporación Universitaria Lasallista.

\section{Toma muestras}

Fueron realizados 122 coprológicos, la muestra fue obtenida por extracción directa de la ampolla rectal, con guantes estériles para evitar contaminación y lubricación con aceite mineral, se recolectaron unos 80 gr aproximadamente por animal, los cuales fueron preservados en recipiente plástico para coprológicos refrigerados a $3^{\circ} \mathrm{C}$ en promedio y llevados al laboratorio de diagnóstico clínico veterinaria "Hermano. Marco
Antonio Serna, f.s.c, de la Corporación Universitaria Lasallista, Caldas, Colombia.

Las muestras fueron procesadas individualmente por método de frotis directo y luego sometidas a flotación solución salina saturada (Técnica: Willys Molloy).

\section{Evaluación fecal}

Las heces evaluadas fueron sometidas a escala cualitativa de evaluación de la presencia de trofozoitos y quistes de Giardia duodenalis, bajo la metodología de cruces, dando valores de 0 (Sin presencia), 1 (Baja), 2 (Moderada), 3 (Alta), (Figura 1 y Figura 2).

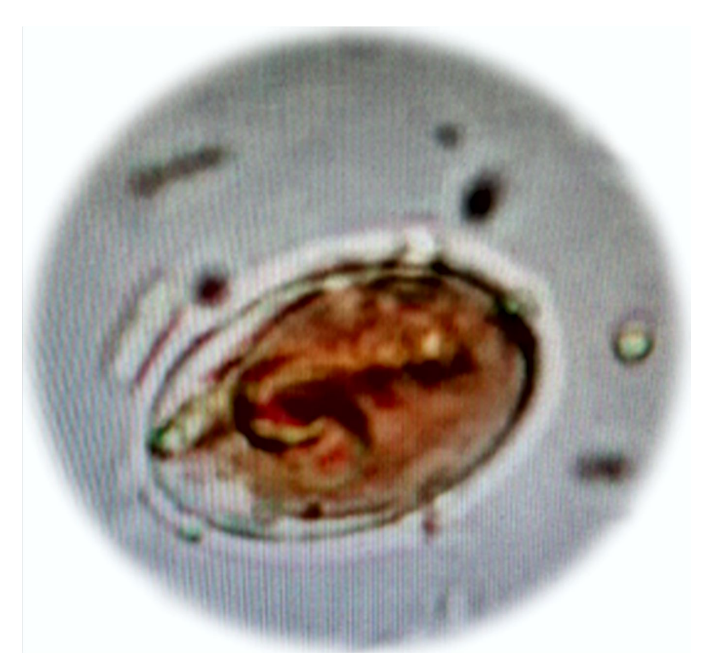

Figura 1. Microfotografía a $40 \mathrm{X}$ de quiste de Giardia duodenalis

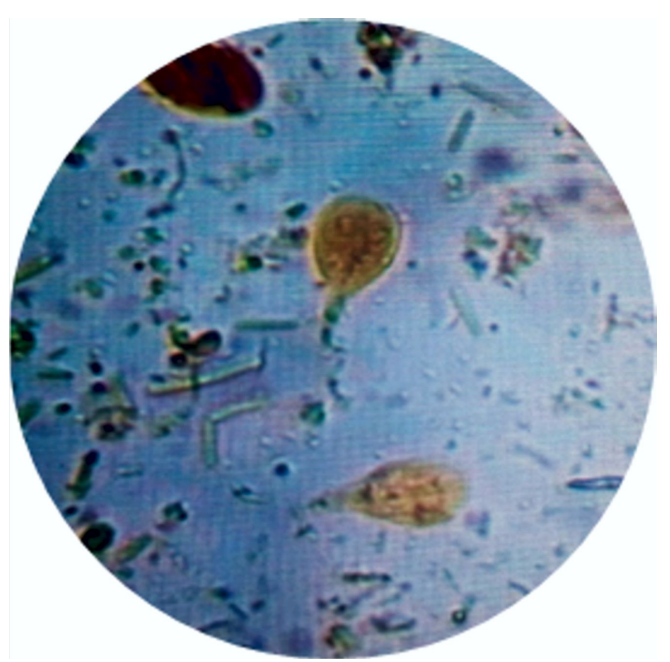

Figura 2. Microfotografía a $40 \mathrm{X}$ de trofozoíto de Giardia duodenalis 


\section{Análisis estadístico}

Los resultados fueron tabulados en programa Excel $\mathbb{R}$ (Microsoft@, Washington, EUA), luego fueron sometidos a cálculo de promedios aritméticos y valores porcentuales, además de determinación del cálculo de prevalencia de la siguiente forma

$P=$ Número de animales con Giardia duodenalis/Total de la población x 100

\section{Resultados}

De los 112 coprológicos realizados, se encontró que $60(55 \%)$ presentaron parásitos gastrointestinales de los cuales solo $10(8.9 \%)$, presentaron formas de G. duodenalis, en las heces y correspondiendo este tipo de parásito solo al 16,6\% de la parasitosis total, en cuanto a la presencia de cruces de los animales parasitados, con 1 (una cruz) dos caninos $(20 \%)$, para 2 (dos cruces) cinco caninos (50\%) y con 3 (tres cruces), tres ejemplares $(30 \%)$, mientras que para la presencia de las formas reproductivas del parasito, se estableció, que para los trofozoítos, los hallazgos mostraron que tres coprológicos, tenían 1 cruz, cuatro muestras mostraron 2 cruces y cinco caninos tuvieron 3 cruces respectivamente, mientras que la presencia de quistes, la relación fue para 1 cruz dos caninos, 2 cruces, seis caninos y para 3 cruces, dos muestras.

No hubo diferencia estadística entre la presencia de Giardiasis entre machos y hembras; tampoco se observó diferencia según la edad.

\section{Discusión}

La prevalencia determinada para el presente estudio en el centro de bienestar CEIBA, de Rionegro, Colombia fue de $8.9 \%$, siendo similar a las descripciones dadas por otros autores que oscilan entre $5-12 \%$ aproximadamente en otros centros de bienestar animal (Alarcón et al., 2015; Caraballo et al., 2007; SierraCifuentes et al., 2015), pero es mucho menor a lo que describen Cabrera y Molina (2016) en un albergue con condiciones similares, donde la prevalencia fue $54.5 \%$ al (2015), la diferencia puede atribuirse a la biología de la enfermedad pues se evaluó la presencia del parásitos en dos condiciones medio ambientales muy distintas, mientras que Rionegro está a 2080 msnm, Caldas esta 1750 msnm, lo cual puede influir por las condiciones de temperatura en la prevalencia del parásito, desde la biología del agente (Adam, 2001; Caccio et al., 2005), debido a que condiciones climáticas y calidad del agua, pueden jugar un papel importante en la epidemiología de la enfermedad, lo cual se puede corroborar en la forma de transmisión en pacientes humanos (Rodríguez et al., 2014). Los hallazgos encontrados son alentadores, en especial si se comparan con otros resultados en países desarrollados donde la prevalencia en se encuentra entre $10-21 \%$, pero debe ser claro que otros países usan pruebas inmunológicas para el diagnóstico, lo cual garantiza mayor sensibilidad (Asano et al., 1991; Olson, 2010; Pallant, 2015). Estos resultados pueden indicar no solo que se están dando buenas medidas de control del parásito, sino que las condiciones ambientales pueden ser desfavorables para el mismo (Adam, 2001; Payne y Artzer, 2009).

En cuanto al análisis de los resultados de las formas parasitarias de G. duodenalis en la presente descripción, se pudo establecer que la forma quística es la más prevalente con dos cruces, lo que indica que hay una alta posibilidad de reinfestación de los animales y la alta presencia de formas trofozoíticas, demuestran que los caninos están reinfectándose constantemente en el albergue y convirtiéndose en un riesgo para la salud pública, esto puede explicar que los animales presentan un equilibrio epizoótico en la zona, pero no descarta el riesgo zoonótico para el personal y habitantes de la zona, por el manejo de excretas con presencia de quistes (Pallant, 2015; Thompson, 2000, 2004); no solo porque la giardiasis se comporta como uno de los parásitos gastrointestinales más comunes a nivel mundial (Bouzid et al., 2015), sino que a la vez encarna un factor de riesgo en la salud humana (Botero y Restrepo 2012; Esch, y Petersen, 2013; Torres et al., 2011), explicado claramente en muchos estudios don los caninos resultan ser fuente de contagio para los humanos (Thompson, 2000), dado por la capacidad infecciosa que tiene varias variedades de Giardia spp (Fava et al., 2016; Lalle et al., 2005; Pallant., 2015; Thompson, 2000).

La ausencia de estratificación de sexo y edad se debe a que estudios previos hechos por Alarcón et al (2015), Cabrera y Molina (2016), Caraballo et al., (2007), Sierra-Cifuentes et al., (2015) y Montoya y Roldan (2007), no pudieron establecer que exista una relación entre sexo y edad con respecto a la infestación con giardia, tampoco se tuvo en cuenta las condiciones de raza, pues el $100 \%$ de los ejemplares del centro de bienestar son de raza mestiza (cruces) (Cabrera et al., 2003), aunque las descripciones de autores como Huamancayo (2015), sugieren que los animales menores de un año son más susceptibles.

Es importante resaltar que la G. duodenalis tiene la capacidad, de que los quistes al ser excretados en las heces de forma intermitente, puede llevar a que se reporte falsos negativos, esto indicaría que en algún 
momento la prevalencia en el centro de bienestar sea mayor, convirtiéndose en un potencial zoonótico (Itoh et al., 2009).

\section{Conclusión}

La prevalencia en el centro de bienestar animal CEIBA, de Rionegro, Antioquia es baja con respecto a las descripciones de otros autores, posiblemente las condiciones medio ambientales y las prácticas sanitarias en los caninos del centro sean las responsables del bajo nivel de la G. duodenalis en el centro.

\section{Agradecimiento}

Al personal del Centro de Bienestar Animal "CEIBA", a la Alcaldía de Rionegro y al personal administrativo de la clínica "Hermano Octavio Martínez López, f.s.c", Doctora Marcela Valencia por su disposición y apoyo en la labor académica e investigativa.

\section{Referencias}

Adam R. Biology of Giardia lamblia. Clin Microbiol Rev. 2001; 14(3):447-475.

Alarcón ZK, Juyo V, Larrotta JA. Caracterización epidemiológica de parásitos gastrointestinales zoonóticos en caninos con dueño del área urbana del municipio de La Mesa, Cundinamarca. Rev. vet. y zootecnia. 2015;62(1):20-36.

Asano R, Hokari S, Murasugi E, Arracima Y, Kubo N, Kawano K. Studies on the

Giardiasis as the zoonosis II. Giardiasis in dogs and cats. Kansenshogaku Zasshi. 1991;65:157-161.

Botero D, Restrepo M. 2012. Parasitosis humanas. 5ta edición. Medellín: Corporación para investigaciones biológicas. 93 p.

Bouzid M, et al. The prevalence of Giardia infection in dogs and cats, a systematic review and meta-analysis of prevalence studies from stool samples. Veterinary Parasitology. 2015;207(34):181-202.

Caccio S, et al. Unravel-ling Cryptosporidium and Giardia epidemiology. Trends Parasitol. 2005;21:430-437.

Cabrera, GA; Molina Díaz, VM. Prevalence of Giardia duodenalis in a canine shelter in Caldas, Antioquia (2015). Journal of Agriculture and Animal Science. 2016;5(2):70-80.

Cabrera P, Ordóñez OE, Cortés JA, Rodríguez J, Villamil LC. Determinación de parásitos zoonóticos (helmintos y protozoarios) en caninos del Centro de Zoonosis de Bogotá, D.C. Biomédica. 2003;23:153.

Caraballo A, Jaramillo A, Loaiza J. Prevalence of parasitic intestinal in canineattended in the veterinary and animal production center of the CES University. Rev MVZ CES, 2007;2(2):24-31.
Cardona E, et al. Comparación de métodos convencionales y moleculares para la detección de Giardia lamblia en heces humanas. Luna Azul. 2014;38:159-170.

Esch KJ, Petersen CA. Transmission and epidemiology of zoonotic protozoal diseases of companion animals. Clinical microbiology reviews. 2013;26(1):58-85

Fava NM, Soares RM, Scalia LA, da Cunha MJ, Faria ES, Cury MC. Molecular typing of canine Giardia duodenalis isolates from Minas Gerais, Brazil. Experimental parasitology. 2016;161:1-5.

Ferraz IC, Chagas da Silva ME, Berendonk-Handam N, Leal-Alencar $M$, Sotero-Martins A, et al. Socioenvironmental conditions and intestinal parasitic infections in Brazilian urban slums: a crosssectional study. Rev. Inst. Med. trop. S. Paulo. 2017;59:1-10.

Fernandes de Mendonça - Uchôa F, Pittella - Sudré A, de Barros Macieira D, Pereira - Almosny NR. The influence of serial fecal sampling on the diagnosis of giardiasis in humans, dogs, and cats. Rev Inst Med Trop São Paulo. 2017;59:e61

Geurden - Thomas PH, Sarrea - Charlotte DL, Vercruyssea - Jozef CE. The efficacy of a treatment with fenbendazole against an experimental Giardia duodenalis infection in lambs. Small Ruminant Research. 2011;96:211-215.

Huamancayo F, Chávez A. Giardiasis en Perros Menores de Tres Años que Concurren a los Parques Públicos del Distrito de Santiago de Surco en Lima Metropolitana. Rev.Invest.Vet. del Perú. 2015;26(2):296-302.

Itoh N, Kanai K, Hori Y, Hoshi F, Higuchi S. Prevalence of Giardia intestinalis and other zoonotic intestinal parasites in private household dogs of the Hachinohe area in Aomori prefecture, Japan in 1997, 2002 and 2007. J Vet Sci. 2009;10(4):305-308.

Ivanov I. Giardia and giardiasis. Bulgarian Journal of Veterinary Medicine. 2010;13(2):65-80.

Jiménez-Albarrán M, Odda R. Coprological Study on Intestinal Ihfection in Northern Morocco (Provinces of Tangier, Tetuan and Larache ). Rev San Hug Púb. 1994;68:1980-1981.

Kotloff K. et al. Burden and aetiology of diarrhoeal disease ininfants and young children in developing countries (the Global Enteric Multicenter Study, GEMS): a prospective, case-control study. Lancet. 2013;382:209-222.

Lalle M, Pozio E, Capelli G, Bruschi F, Crotti D, Cacciò S. Genetic heterogeneity at the -giardin locus among human and animal isolates of Giardia duodenalis and identification of potentially zoonotic subgenotypes. Int J Parasitol. 2005;35(2):207-13.

Meireles P, Montiani-Ferreira F, Thomaz-Soccol V. Survey of giardiasis in household and shelter dogs from metropolitan areas of Curitiba, Parana state, Southern Brazil. Vet Parasitol. 2008;152(34):242-248.

Montoya LM, Roldan LM. 2007. Prevalencia de giardiasis en perros de Medellín con un laboratorio de referencia. [Tesis de maestría].[Medellín, Colombia]. Universidad CES.

O'Handleya RM, Buretb AG, McAllisterc TA, Jelinskid M, Olson ME. Giardiasis in dairy calves: effects of fenbendazole treatment on intestinal structure and function International. J Parasitology. 2001;31:73-79. 
Oliveira T, Amarante A, Ferrari T, Nunes L. Prevalence of intestinal parasites in dogs from Sao Paulo State, Brasil. Vet Parasitol. 2002;103:19-27.

Olson M, Leonard N, Strout J. Prevalence and diagnosis of Giardia infection in dogs and cats using a fecal antigen test and fecal smear. Brief Communication CVJ. 2010;51:640-642.

Pallant L, Barutzki D, Schaper R, Thompson RA. The epidemiology of infections with Giardia species and genotypes in well cared for dogs and cats in Germany. Parasites \& vectors. 2015;8(1):1-14.

Payne PA, Artzer M. The Biology and Control of Giardia spp and Tritrichomonas foetus. Vet Clin North Am Small Anim Pract. 2009;39(6):993-1007.

Rodríguez R, Cob L, Domínguez J. Frecuencia de parásitos gastrointestinales en animales domésticos diagnosticados en Yucatán, México. Rev Biomed. 2011;12:19-25.
Rodríguez V, Espinosa O, Carranza JC, Duque S, Arévalo A, Clavijo JA, Urrea DA, Vallejo GA. Genotipos de Giardia duodenalis en muestras de niños de las guarderías del Instituto Colombiano de Bienestar Familiar y de perros en Ibagué, Colombia. Biomédica. 2014;34:271-281.

Sierra-Cifuentes $\mathrm{V}$, et al. Prevalencia de parásitos intestinales en perros de dos centros de bienestar animal de Medellín y el oriente antioqueño (Colombia), 2014. Rev. Med. Vet. 2015;30:55-66.

Thompson R. Giardiasis as a re-emerging infectious disease and its zoonotic potential. Int J Parasitol. 2000;30(12):1259-1267.

Thompson R. The zoonotic significance and molecular epidemiology of Giardia and giardiasis. Vet Parasitol. 2004;126:15-35.

Torres G, Zapata M, Restrepo M, Ríos L. Investigación científica sobre genotipificación y distribución de Giardia intestinalis en humanos y caninos de América. Rev Salud Uninorte. $2011 ; 27(1): 49-62$

Vanesa Arenas: https://orcid.org/0000-0002-0044-4950

Víctor Molina: https://orcid.org/0000-0002-6839-6481 